\title{
Affordable Mobile technology towards Preventive Health care: Rural India
}

\author{
Dr. Susan Thomas \\ Ex -Professor of Public health Dentistry,NIMS Dental College,NIMSUniversity,Jaipur,India
}

\begin{abstract}
Most of the chronic non- communicable diseases are consequences of unhealthy life style practices, resulting in premature morbidity or mortality. The cost of treating chronic diseases has increased the economic and social burden nationally as well as globally. In developing countries such as India, approximately $3 / 4$ of the population reside in rural areas experiencing inequalities in health care access.

Mobile phone usage has increased globally. These devices are commonly used by the community and are affordable to all segments, especially the rural population. Innovative mobile health technology with publicprivate partnership can sensitize customers on various health products and services by mobile apps or text messages.Empowered by many preventive health care programs, the $m$ - health text message technology becomes a potential powerful tool for behaviour change as it is widely available and inexpensive. By improving the health-care seeking behaviour and treatment compliance, the efficiency of treatment could increase thus reducing the costs to health plans. This paper discusses the implication of mobile phone messaging to improve the process of health care delivery and health service. Through increase preventive care use, today's patients and community health workers can make better choices to successfully modify their behaviour and become healthy and productive citizens.
\end{abstract}

Keywords: Affordable,Behaviour change,Mobile text message, Preventive health service, Rural India

\subsection{Health care in India}

\section{Introduction}

India is a young nation in which an estimated 65 per centof the population of 1.2 billion is under the age of 35 . Among this group 460 million people are between the ages of 15-35. Since the Indian population is so skewed towards the young, the country must invest in their education and health care in order to ensure their employment in productive work, thereby boosting the economy.

In India the distribution of wealth and the capacity to generate income could be represented in the form of an economic pyramid. At the top of the pyramid are the wealthy with numerous opportunities for generating income. More than 800 million represent the bottom of the pyramid who live on less than $2 \$$ per day.[1]These people represent the forgotten India and are rarely talked about.

Government spending on health care in India is less than $2 \%$ of the GDP which is among the lowest worldwide, although it is high when compared to other developing nations. Recently India's private health insurance industry showed an annual growth of 35\%; however $85 \%$ of the population remains uninsured.

In 2001, 28 per cent of India's people lived in urban areas. The majority of the Indian population lived in 600,000 villages and over half of these villages were not easily accessible due to poor roads. In contrast $80 \%$ of the trained human resources were concentrated in urban areas showing a gross disparity in health care accessibility between the urban and rural population.

Many of the traditional practices in India are detrimental to the health and even to the status of women. The local beliefs and culture influence health and many related aspects of human behaviour such as help seeking, treatment compliance and patient satisfaction which prevent some of the rural groups from accessing health care benefits.

The geographical inaccessibility, unavailability of affordable health care and reluctance to seek medical assistance for India's billion-plus people present enormous challenges and opportunities for the medical community, insurers and other service providers.

Today's health-care technology that is used for communication and treating people is amazing.Mhealth is one aspect of e-health which is used for practice of medicine and public health, supported by mobile devices.[2] M- health devices such as mobile phones, tablets,computers and PDA's are used for health services as well as information. The term m-health was coined by Professor RobertIstepanian for the use of 'Emerging mobile communication'.

This paper addresses how mobile wireless health technology could contribute to improvement of preventive health care practices, especially inthe remotest and most undeservedpopulation, irrespective of place or social class. 


\section{The Burden of Chronic Non-communicable Diseases (CNCD) andits Economic impact- India}

NCDs kill about twice as many people in India as communicable diseases. The chronic diseases have emerged as a critical public health problem in India. Chronic diseases affect health and quality of life resulting in illness, disability, extended pain and suffering, and major limitations in daily living. They also have economic and social consequences. With a limited infrastructure for chronic disease care, India is ill-equipped to deal with the escalating chronic disease epidemic, which has been threatening to affect its economic development in recent decades.

Non-communicable diseases currently account for 35 million out of 58.7 million deaths annually worldwide, and the majority of them happen to be in the low and middle-income countries (28.1 million). In developing countries alone, an estimated 8 million such deaths per year occur in the population which is below 60 years of age, and could potentially beprevented.

Globally, government spending on treatment of NCDs is 60 per cent of the total amount spent on health care, while in India it is 26 per cent. Private out- of- pocket spending by families on CNCD is 44 per cent worldwide, and in India it is 90 per cent.[3]This high cost of treatment in India could potentially affect many newly emerged middle class families in India which are potentially facing poverty.

India's top four chronic diseases are cardiovascular diseases (CVDs), diabetes, chronic obstructive pulmonary disease and cancer. These diseases are responsible for 60 per cent of the world's deaths andof these deaths 80 per cent are happening in the poorest populations of the world.Cardiovascular diseases are the leading cause of death in the age group of 35-64 years in IndiaIn 2000, 9.2 million years of productive life were lost in India. The projected cumulative loss of national income for the country due to the NCD mortality for 2006-2015 is projected to be USD 237 billion.[3]

In 2007, India spent $4.1 \%$ of its gross domestic product on health services or USD40per capita. Unhealthy lifestyles and behaviours (e.g., smoking, excessive alcohol consumption, physical inactivity, and imbalanced diets) are responsible for the growing epidemic of CNCDs. These risk factors could be responsible for high levels of bloodpressure, cholesterol and glucose leading to various CNCD. 40-50\% of NCD related deaths are preventable. Early detection of hypertension and other risk factors reduces cardiovascular disease mortality by $35-60 \%$, andfor cancer by $30-80 \%$.

It is important that India must shift government health expenditures towards prevention, screening, early intervention and medical treatments thus reducing the burden of disease. Costs associated with chronic disease conditions could be avoided if measures to promote healthy life-styles and environments were implemented. These measures are essential for the health of India as well as its economic progress. Governments have to recognize the social determinants and behavioural issues and address these risks in order to provide the services that people are being denied.

\section{Mobile HealthInnovations forSocial Development and Economic transformation}

Over half of the world's 6.5 billion people now use a mobile and over 60 per cent of mobile phone users live in developing countries.[4]Improvements in telecommunications technology infrastructure, reduced costs of mobile handsets, and a general increase in non-food expenditure have influenced this trend. [5] Mobilebased innovations are quickly emerging as the new frontier in transforming government, health, banking, education and many other sectors due to fast growing penetration of mobile phones even in the poorest and remotest areas of the globe. Many services can now be made available on a 24x7x365 basis at any place in the world covered by mobile networks, which today means almost everywhere. Through mobiles, for the first time ever, many public and private services have now reached poor households and communities. The demand for mobile applications is fast picking up in developing countries. A multitude of highly innovative applications have been developed for mobile banking and payments, phone based information services for farmers and fishermen, locations based medical services, monitoring and data collection in the health sector, to name a few. However, the enormous potential of mobile devices for transforming delivery of public and financial services is still largely untapped. $\mathrm{M}$ - Health projects have applications for the developed nations. With the rapid rise of mobile phones in low income nations, various applications for developing countries have emerged. These applications are a means of providing greater access, as well as improving the capacity of health systems to larger segments of the rural population in developing countries.

With the development of innovative health technologies, the bottom-of- the-pyramid consumers get an opportunity to participate and benefit from the choices of health products and services made available through the market mechanism. Having been increasingly sensitized to various health products and services the consumers demand high technology solutions to their problems. Once the bottom-of-the-pyramid consumers get access to innovative technologies, they benefit from wider knowledge resulting in rapid economic and social transformation. 
With the changes of income inequalities, more people at the bottom of the pyramid can aspire to middle class life style. With this increase in the middle class, the pyramid could morph into a diamond reflecting the social and economic transformation.

\section{Strengthening Remote area health services through mobile communications}

Mobile phone penetration is rapidly increasing particularly in the Asia- Pacific region.The usage is rising faster in India than anywhere in the world, with nearly six million mobile users added every year.The proliferation of wireless devices, such as mobile phones, among the poor who represent the bottom of the economic pyramid is universal, with $29.4 \%$ of the rural Indian population having access to a mobile network in 2011. Mobile phones support basic voice and phone message services enabling two way communications and are an important communication globally. Many mobile phones have a camera to capture pictures or short videos that can be viewed on the phone, downloaded to one's computer or transmitted to others.

Most digital mobile phones provide messages as basic SMS(short message service) functions also known as text messaging and MMS (multi-media services).SMS in particular has rapidly developed into a powerful real time communication medium especially among young adults who serve as the backbone on the use of mobile technology.

As compared to other communication channels mobile text messages have the advantage of instant and low cost transmission. The special features such as mobility, direct and instantaneous access, direct communication and easy to call and respond enablethe possibility of using mobile phones for health information. Usage of health text messages through the global public-private partnership is linking more people globally, even in the most remote villages.Literature reviews on the use of mobile phones in health promotion and preventive care has demonstrated wide applications and potential use. Basic text messaging has helped to enhance efficiency of health services, improve diagnosis, treatment and rehabilitation, provide appointment reminders, improve patient compliance with medications and monitor chronic conditions. In rural areas of the developing world where many new diseasesout-break occur and in a disaster situationmobile phone is often a community's only link to the outside world.Those that have enrolled in the text message plans, such as health promotion in smoking cessation for example, choose their designed program and receive periodic health messages.

Voxiva's technology turns a village mobile phone into a communication device on par with that of a computer.Voxiva was co- founded by Paul Meyer in March 2001.[1]It helps to deliver practical technology solutions through the reach of software applications to people and communities who do not have internet access. In this way Voxiva helps to promote health and brings significant social value in the developing world.

Providing support and education to remote-area practitioners via information technology (IT) is a new and emerging field. Maintenance of professional competence is a key area of concern for health providers in remote areas who experience difficulty in accessing current research, guidelines and educational updates. The rural health professionals are able to send and receive voice messages even if they do not own a land telephone.Users are also able to receive health alerts,information about diseases, natural disasters, vaccination programs, and also training opportunities.

Some possible challenges in using mobile phone messaging includes the risk of inaccurate data input ,lack of understanding or misinterpretation of the information ,difficulties in reading for those with poor vision or problems with literacy. Failures or delays in message delivery are rare but possible. Incomplete coverage of mobile networks as well as information overload are some concerns to be addressed.

\section{Community participation through mobile text messages (wellness program)}

Many chronic conditions which result from health-risky behaviours are placing an increasing burden on the health systems around the world. Life-style factors such as improper diet, lack of exercise, tobacco use and obesity share a major burden of health care cost.

The innovations and explosion of mobile devices have changed the way people could communicate to access personal and reliable health information. Voice calling, data entry and data storage and SMS provide effective and efficient tools which support the communication needs of behaviour changed interventions. Health information on prevention and treatment can be distributed via text message or mobile internet connections. Mobile Short Message Services (SMS) has high penetration in the lower socio-demographic population with poorer health.[6] Personally tailored messages are more effective for health behaviour change.Since phones are often accessible by only a single individual, outreach for sensitive medical issues, such asreminders for medical appointments or information on lab results, could be kept confidential.

\section{A few examples of the effective use of SMS in health care are noted below:}

Daily and weekly SMS sent to user's mobile phones with type 1 diabetics mellitus have resulted infavourable changes in the users in diabetes self- efficacy and adherence to treatment.[7,8] 
A community based anti-obesity program by Patrick et al[9]used mobile phone short message services messaging. The results of their study showed SMS messaging is a productive channel of communication to promote behaviour modification in weight control and anti-obesity health education programs.

Studies have shown that SMS messages have made possible more timely diagnoses and treatment of chlamydia trichromatic infection, thus reducing costs for staff follow up.

Favourable results have been observed in text message based intervention which offers potential for a new way to help young smokers to quit.[10]Thisintervention program is affordable, age appropriate and not location dependent.

Immunization reminders delivered by text message intervention have shown promising results. More than half of the parents in a study were willing to register their cell phone numbers for future immunization messaging via cell phone calls or text messages.[11, 12]

The use of cell phones and text messaging interventions in improving health outcomes (asthma symptoms, stress levels, smoking quit rates) resulted in significant improvements in compliance with medicine taking, disease monitoring and management and education.[13]

Failure to attend health care appointments has a significant impact not only on the health of the patients but also on the health system costs. This could lead to delays in diagnosis and appropriate treatment. Mobile phone messaging applications such as SMS and Multimedia Service could provide an important, inexpensive delivery medium for healthcare appointment reminders.[14]Text messages on medication reminders and appointment dates have been a source of self-health care, especially for the elderly.

Beyond capabilities for voice and text messages, health related applications for mobile phones have emerged in the commercial sector. Some mobile phone applications helpusers track their behaviour related to weight management. Behaviours such as calorie counting can be supported by software on the phone. Reminders for special dietary needs could be pre-set and then sent to the user.[7] Empowered with various health apps or free motivational and wellness related health tips via text messages, the active consumers stay healthy and are able to maintaina diet controlled in sugar, salt and saturated fat, count calories, manageweight, monitorexercise, quitsmoking, handlestress, use seat belts or helmets and stay alcohol free, especially while driving.

Innovative application helps to improve nurse-patient communication and monitor health outcomes in chronic diseases. These technologies are expected to increase overtime in community management of cancer, heart disease, asthma and diabetes. In resource-limited settings malaria control programs should consider use of text messaging to improve the case management practices of health workers.

The privilege of obtaining timely health information and health service choices irrespective of the social class and place at a very minimal cost is a boon to the health industry.

\section{Conclusion}

Affordable preventive health care can be accessible to the most deserving rural population especially in developing countries. With the rise in mobile phone usage globally, today's patients and community health workers even in the remotest rural areas can be empowered with various preventive health care programs through innovative m-health text messages or mobile apps enabling them to modify unhealthy life style practices which are mainly responsible for major chronic non-communicable diseases. Sensitising the customers with health care products and services helps them to demand for better health services andreduce the gap in health inequalities which exists especially among the rural population. Behaviour change increases the efficiency of treatment and outcomes for providers, thus reducing costs to health plans through increased utilisation of preventive care. This way the community can produce youth who are healthy and productive, thus reducing the economic and social burden of the country.

\section{References:}

[1]. Prahalad, C.K The market at the bottom of the pyramid.The fortune at the Bottom of the pyramid. Pearson education ,inc.2005,3114

[2.] R.Istepanian.Engineering in medicine and biology society.Proceedings of the 25th annual international conference of the IEEE; 2;2003,1414-16

[3]. AMahal.,AKaran .andM Engelgare, .The economic implications of non-communicable disease for India.Health,Nutrition and population (HNP)discussion paper.

[4.] KKalba,. The Adoption of Mobile Phones in Emerging Markets: Global Diffusion and the Rural Challenge. International Journal of Communication(2)2008,631-661.

[5]. Economist leaders. The limits of leap frogging, technology and development. The Economist, 2008.

[6]. B.S. Fjeldsoe, A. L. Marshall, and Y. D. Miller. Behavior Change Interventions Delivered by Mobile Telephone Short-Message Service. Am J Prev Med;36(2):2009, 165-173

[7]. H.S Kim, H.S Jeong. A nurse short message service by cellular phone in type-2 diabetic patients for six months.Journal of Clinical Nursing; 16, (6) , 2007,1082-1087,

[9]. K. Patrick, 囚F. Raab, M. A Adams, L.Dillon, M. Zabinski, et al. Text Message-Based Intervention for Weight Loss: Randomized Controlled Trial. J Med Internet Res.; 11(1):2009 
[10]. A. Rodgers, T. Corbett, D.Bramley, T. Riddell, M. Wills, R.B. Lin, M. Jones. Do u smoke after txt? Results of arandomized trial of smoking cessation using mobile.Tobacco Control 14:;2005,255-261

[11]. J. Jamison, S. Sutoon, H. Gilbert.Delivering Tailored Smoking Cessation Support via Mobile Phone Text Messaging: A Feasibility and Acceptability Evaluation of the Quit text Program. Journal of Applied Bio behavioral Research; 17 (1), : 2012,38-58.

[12]. S. J. Clark, A. Butchart, A. A. Kennedy, K. J. Dombkowski.Parents' Experiences With and Preferences for Immunization Reminder/Recall Technologies.Pediatrics; 128 (5): 2011

[13]. S. Krishna, S. A. Boren, E. A. Balas.Healthcare via Cell Phones: A Systematic Review.Telemedicine and e-Health. 15( 3): 2009,231-240.

[14]. Car J., Gurol-UrganciI, De Jongh, T., Vodopivec-Jamsek, V., Atun, R. Mobile phone messaging reminders for attendance at Health care appointments. Cochrane review, The Cochrane Library(7) 2012.http://www.thecochranelibrary.com 